\title{
Self-organized phase-transition lithography for all-inorganic photonic textures
}

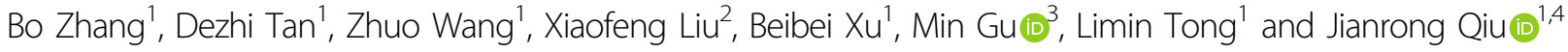

\begin{abstract}
Realizing general processing applicable to various materials by one basic tool has long been considered a distant dream. Fortunately, ultrafast laser-matter interaction has emerged as a highly universal platform with unprecedented optical phenomena and provided implementation paths for advanced manufacturing with novel functionalities. Here, we report the establishment of a three-dimensional (3D) focal-area interference field actively induced by a single ultrafast laser in transparent dielectrics. Relying on this, we demonstrate a radically new approach of self-organized phase-transition lithography (SOPTL) to achieve super-resolution construction of embedded all-inorganic photonic textures with extremely high efficiency. The generated textures exhibit a tunable photonic bandgap (PBG) in a wide range from $\sim 1.3$ to $\sim 2 \mu \mathrm{m}$. More complicated interlaced textures with adjustable structural features can be fabricated within a few seconds, which is not attainable with any other conventional techniques. Evidence suggests that the SOPTL is extendable to more than one material system. This study augments light-matter interaction physics, offers a promising approach for constructing robust photonic devices, and opens up a new research direction in advanced lithography.
\end{abstract}

\section{Introduction}

Interference phenomena, ubiquitous in light-light and light-matter interactions, allow for manipulation of the distributions of the electromagnetic field and manufacturing of functional structures ${ }^{1-5}$. Some of the wellknown examples include multibeam interference ${ }^{1,6}$, holographic interference ${ }^{7,8}$, surface-plasmon polariton (SPP) interference ${ }^{9,10}$, and interference between the incident light and scattered waves from defects or inhomogeneities $^{11,12}$, enabling patterning periodic structures with high efficiency and throughput. Even though various interference fields have been considered as wellestablished processing tools on the surface, the 3D spatial interference lithography inside inorganic transparent

\footnotetext{
Correspondence: Dezhi Tan (wctdz@zju.edu.cn) or

Jianrong Qiu (qjr@zju.edu.cn)

${ }^{1}$ State Key Laboratory of Modern Optical Instrumentation, College of Optical

Science and Engineering, Zhejiang University, 310027 Hangzhou, China

${ }^{2}$ School of Materials Science and Engineering, Zhejiang University, 310027

Hangzhou, China

Full list of author information is available at the end of the article

These authors contributed equally: Bo Zhang, Dezhi Tan
}

dielectrics, however, remains very difficult or even impossible without the use of complex beam-shaping manipulation or photosensitizers. Therefore, robust optical elements and embedded photonic integration are lacking. Ultrafast laser-matter interaction has been considered a treasure trove for a variety of novel physical phenomena that may give solutions to current "impossible tasks"13-17. Theoretically, an intense spatial interference field can be actively excited by single-beam ultrafast laser irradiation and may possess the ability of nonlinear material modification. This provides a potentially flexible and high-efficiency shortcut that can quickly generate 3D super-resolution interference lithography in transparent dielectrics without any additional setups or photosensitive components. However, as a pioneering scenario that has not been experimentally demonstrated, its principle and feasibility remain unclarified.

Photonic crystals (PCs), which play an immense role in photon-control engineering ${ }^{18-21}$, are constructed by periodically organized artificial textures with a feature size comparable to the light wavelength ${ }^{22-24}$. In particular,

\section{(c) The Author(s) 2021}

(c) (i) Open Access This article is licensed under a Creative Commons Attribution 4.0 International License, which permits use, sharing, adaptation, distribution and reproduction cc) in any medium or format, as long as you give appropriate credit to the original author(s) and the source, provide a link to the Creative Commons license, and indicate if changes were made. The images or other third party material in this article are included in the article's Creative Commons license, unless indicated otherwise in a credit line to the material. If material is not included in the article's Creative Commons license and your intended use is not permitted by statutory regulation or exceeds the permitted use, you will need to obtain permission directly from the copyright holder. To view a copy of this license, visit http://creativecommons.org/licenses/by/4.0/. 
creating near-infrared light-responsive textures is highly valuable for a wide scope of research fields like optical communication, computing, and sensing ${ }^{25-27}$. However, despite more than 30 years of research and numerous publications on photonic textures by processing photoresists $^{28,29}$, semiconductors ${ }^{30,31}$, and metals ${ }^{32,33}$, there is no effective method that has been established to massively produce all-inorganic photonic textures using a singlestep process. This is one of the greatest limiting factors to the further development of PCs. Besides, achieving photonic textures having a tunable PBG inside transparent dielectrics remains a major challenge for current research and an important component that is missing in advanced nanostructuring.

We recently discovered an unprecedented 3D interference field actively excited by ultrafast laser-matter interaction in $\mathrm{La}_{2} \mathrm{O}_{3}-\mathrm{Ta}_{2} \mathrm{O}_{5}-\mathrm{Nb}_{2} \mathrm{O}_{5}$ glass (LTN glass) that allows the realization of super-resolution lithography of highly regular embedded crystalline photonic textures with tunable optical performances in the near-infrared region. Based on this, a series of photonic structures can be created quickly with a simple one-step process, and the method has been demonstrated to possess a considerable degree of universality in different transparent dielectrics, from glasses to crystals. The problem of high-efficiency construction of complicated all-inorganic 3D photonic elements in transparent dielectrics is resolved.

Here, we present a unique 3D interference phenomenon between the focused ultrafast laser and scattered spherical wave based on the light-matter interaction in the glass to give a precise description of the interference field distribution with a single scattering center model (Supplementary Fig. S2). By exploiting this principle, a strategy of ultrafast laser-induced SOPTL is established to efficiently create large-scale periodic photonic textures (Supplementary Movie S1). In our design, ultrafast laser focusing in transparent media, under proper conditions, can produce a scattering center in the focal area due to the strong multiphoton ionization caused by local modification ${ }^{34,35}$, making it possible to establish an intense scattered light field. The spatial interference in three-dimension between the incident and scattered wave occurs, creating a holographic light field (Fig. 1a). In the glass, this triggers periodic crystallization with the beam-writing process and enables regularly patterned crystal precipitation in 3D along the writing path (Fig. 1b, c). By manipulating the focal light field in the SOPTL, more complex photonic textures can be created with remarkable high efficiency (Fig. 1d). More importantly, an obvious PBG at communication band is produced, which relies on the large refractive index contrast between the crystal phase and the glass matrix, and the PBG is tunable in an extremely wide range $(\sim 700 \mathrm{~nm})$ through the engineering of a secondary-phase transition. We believe that with the inception of the SOPTL concept, more efficient methods and functional materials aimed at large-scale production of all-inorganic photonic textures will be developed in the near future, and present a revolutionary approach to develop embedded photonic devices in transparent media.

\section{Results}

Figure 2a demonstrates the theoretical spatial distribution of the light interference field represented as a series of equal-phase surfaces (EPSs) for constructive interference. When the ultrafast laser beam moves relative to the sample, specific EPSs participate in the SOPTL process due to the limitation of the crystallization condition, leading to the formation of periodic structures. Figure $2 b$ shows the theoretical morphology of the periodic structures in the XZ plane created by the SOPTL (more details about the theoretical derivation and experimental evidence are found in the supplementary information). As expected, the experimentally observed periodic structures, revealed by the intersecting patterns in view planes $(X Z$, $X Y$, and $Y Z$ plane), are consistent with the theoretical results, which confirms the validity of our proposal.

Figure 2c illustrates the curve extracted from the edge of the $X Z$ projection of an equal-phase face, which corresponds to the pattern of the experimentally observed periodic arrays in Fig. 2f. Notably, the interference order of the equal-phase face derived here is as high as 20 , which is the root cause of the inclination of the periodic structures in the $\mathrm{XZ}$ plane. Meanwhile, from the $X Y$ view of the generated textures, arcuate fringes that are similar to the calculated EPSs are observed (Fig. 2d, g). As expected, such arcuate fringes exhibit an obvious dependence on the laser scanning direction, strongly supporting the concept that only EPSs with certain interference orders along the specific orientation participate in the formation of the periodic structure. Furthermore, as a result of the periodic arrangement of EPSs along the beam-writing path, the $Y Z$ view of the textures is theoretically characterized by a series of arcuate fringes along the laser propagation direction (Fig. 2e), in line with experimental observation (Fig. 2h).

The theoretical dependence of the periods at different depths along the $Z$ axis on the interference order is clarified in Fig. 2i, which indicates that the period of the texture varies with the interference order, and also increases with the depth along the laser propagation direction. Figure $2 j$ shows the theoretical relationship between the period and depth with the interference order set as 20, coinciding with actual values (purple points), which further points to the effective EPSs responsible for the SOPTL process. Based on the theoretical model, the radius of the interference fringes gradually increases with depth, inevitably leading to a smaller bending degree of the periodic stripes in the $X Y$ plane, which is in line with 


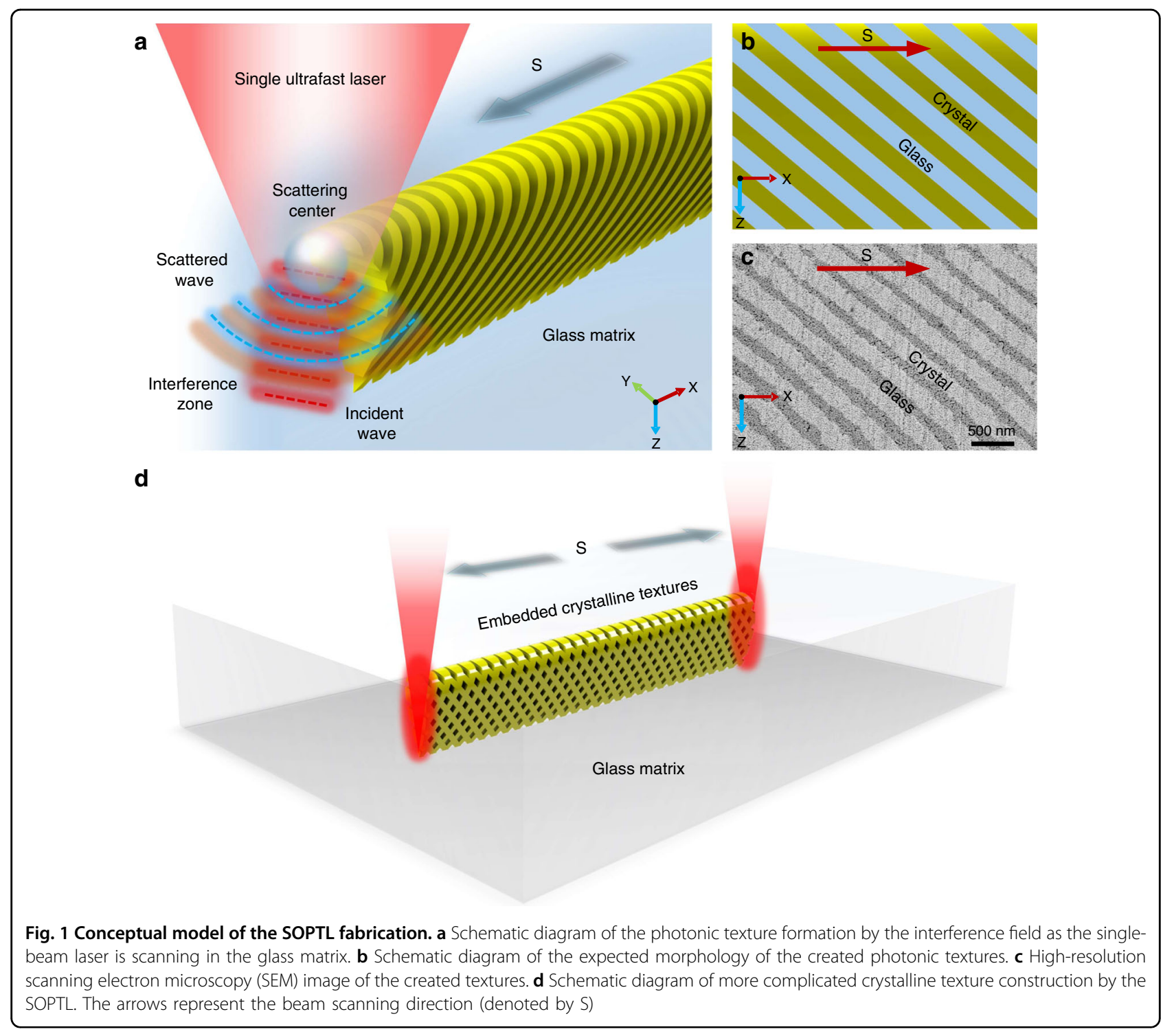

experimental results (Fig. 2k). The simulation indicates that all the EPSs merge in the original focal plane at the depth of zero, meaning that the periodic feature will theoretically disappear. This prediction is verified by SEM observation at the top area of the periodic structures (Fig. 2k, insets). Furthermore, the inclination direction of the periodic structures is dependent on the laser scanning direction and always tilted to the starting point of laser scanning. Supplementary Fig. S3a shows a nearly perfect symmetry between the periodic textures written by scanning the ultrafast laser in opposite directions from the same starting point, which echoes the directiondependent arcuate fringes observed in the $X Y$ view (Fig. 2g). Taken together, both qualitative and quantitative results confirm that a well-defined $3 \mathrm{D}$ light interference is established in the focal area and the SOPTL is triggered to effectively build complex spatial periodic textures.
We presented the fast creation of large-area photonic textures with fine spatial morphology in the glass and demonstrated their phase-transition-based structural properties. As shown in Fig. 3a, the presented lasermodified area, filled with the textures, reaches millimeter scale and the typical writing speed can be as high as $2 \mathrm{~mm} \mathrm{~s}^{-1}$, which shows that the SOPTL possesses a strikingly higher processing efficiency than conventional techniques. Meanwhile, the uniform and intense artificial birefringence signal in the laser-modified area confirms that the SOPTL has a substantial degree of repeatability and robustness against various external perturbations. Figure $3 \mathrm{~b}$ displays an optical image of the periodic structures without mechanical damage, and highly regular nanometer-scale textures embedded in the glass matrix can be observed. This ensures a remarkable optical performance of the created structures. Figure $3 \mathrm{c}$ shows that 


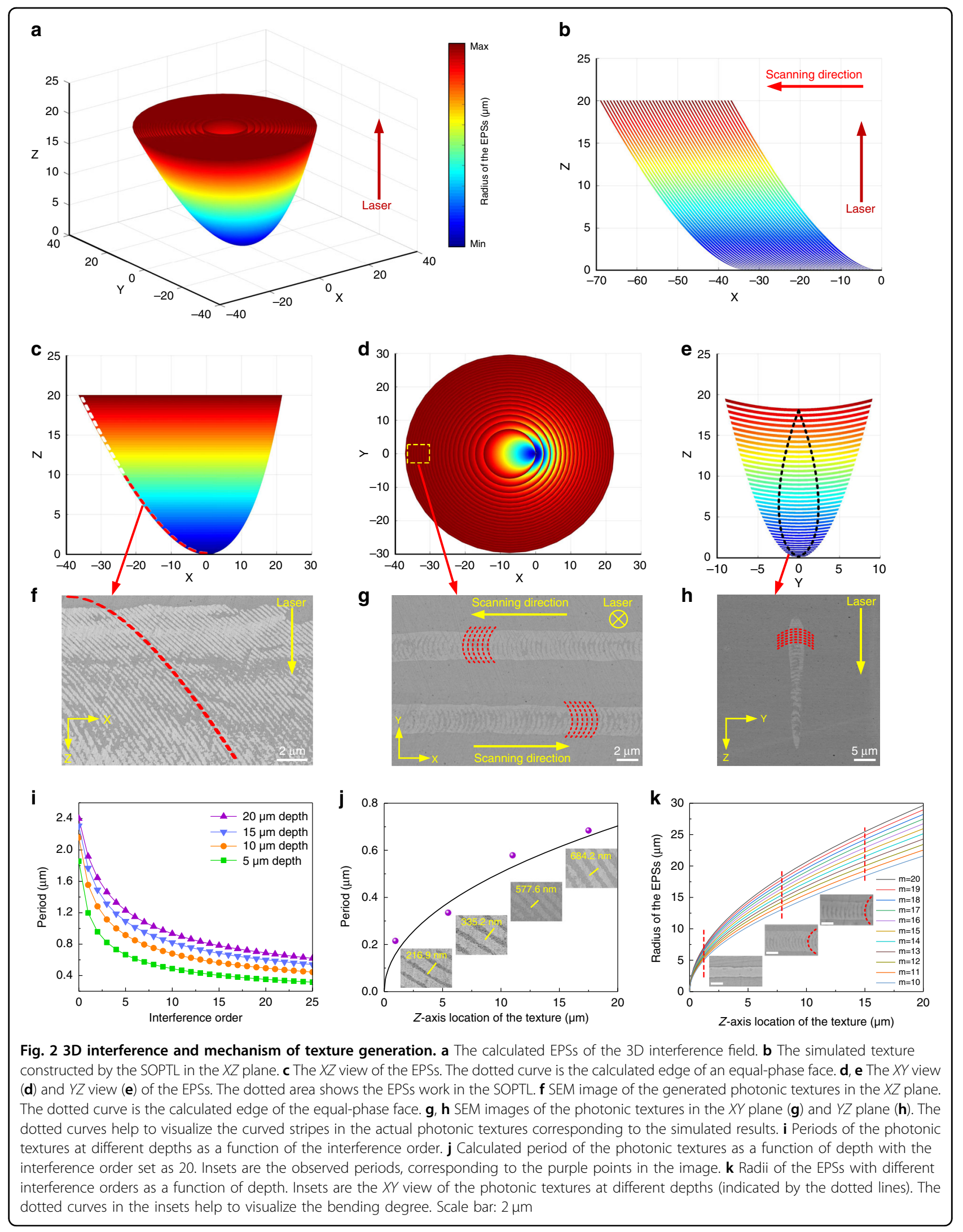



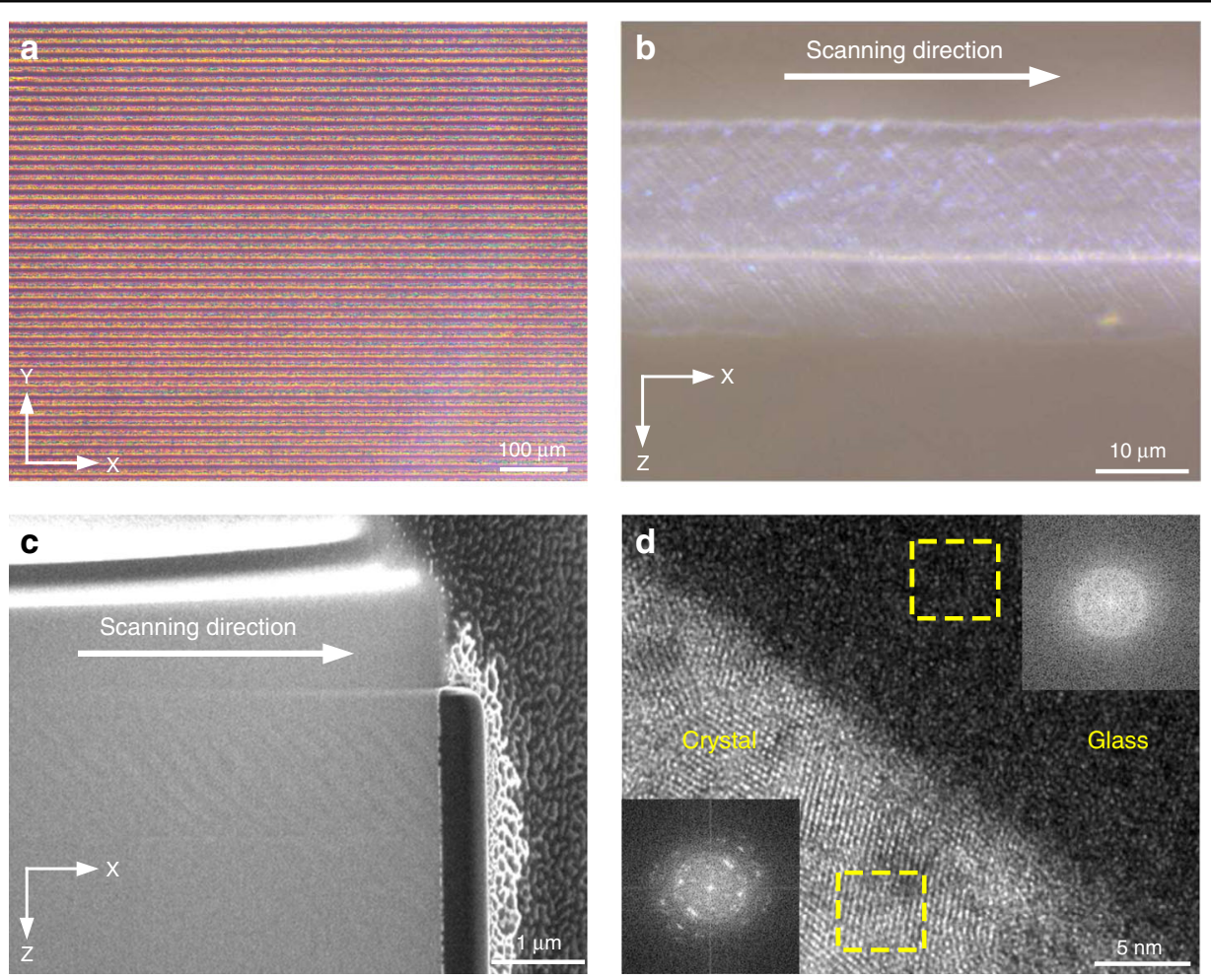

Fig. 3 Structural demonstration of the textures. a Birefringence image of largely produced photonic textures in the glass. $\mathbf{b}$ Optical microscope image of the embedded glass-crystal arrays without any mechanical damage. $\mathbf{c}$ Focused ion beam (FIB) slice of the periodic glass-crystal arrays of the texture. $\mathbf{d}$ HRTEM image of the glass and crystal region of the texture. Insets are the fast Fourier transform (FFT) images of the dotted areas

the created texture is characterized by the presence of periodically assembled bright- and dark-contrast regions along the beam-writing path. High-resolution transmission electron microscopy (HRTEM) confirms that the periodic textures are essentially alternately arranged crystal-glass phases (Fig. 3d), which is in line with the expected products of the SOPTL. The crystallization structure in the textures is also confirmed by the Raman spectra, as shown in Supplementary Fig. S6. Notably, the feature size of the photonic textures is at around $200 \mathrm{~nm}$ (Supplementary Fig. S4a), about $1 / 4$ of the optical diffraction limit $(\sim 800 \mathrm{~nm})$ of the beam-writing system (1030-nm laser and 0.8 NA objective), demonstrating the super-resolution machining capability of the SOPTL. By utilizing the differences in optical properties between the glass and crystal phases, these well-organized textures hold potential as photonic elements.

We have characterized the optical properties of the photonic textures. The probe light with various wavelengths of $980 \mathrm{~nm}$ (Fig. 4a), $1300 \mathrm{~nm} \mathrm{(Fig.} \mathrm{4b),} \mathrm{and}$ $1550 \mathrm{~nm}$ (Fig. 4c) exhibits different transmittance in a sample with multilayer textures along the propagation direction of the writing beam ( $Z$ axis), and the light at $1300 \mathrm{~nm}$ is found forbidden. The transmittance spectrum shows the presence of an obvious broadband transmission reduction in the range of $1000-2000 \mathrm{~nm}$ with a center wavelength at around $1300 \mathrm{~nm}$ (Supplementary Fig. S5a), indicating the appearance of a broad PBG at near-infrared waveband. The periodic distribution of the refractive index in the crystal-glass textures accompanied with the continuously varied period with the depth is responsible for the spectra modulation and broadness. Besides, a polarization-dependent light attenuation effect in the near-infrared region is also observed (Supplementary Fig. S5b). Considering the spatial morphology of the photonic textures (Supplementary Fig. S5c), the binary optical properties can be understood: for the incident probe light along the $Z$ axis, the component propagating perpendicular to the crystalline stripes is modulated by periodic crystal-glass interfaces, and specific wavelengths are selectively reflected (Supplementary Fig. S5d), forming a PBG. Meanwhile, the component that propagates along the crystalline stripes is also affected by the periodic structures. In this case, the periodic crystal-glass arrays are equivalent to the subwavelength gratings (SWGs), which are polarization-sensitive and act as barriers to the incident light with polarization perpendicular to the grating stripes ${ }^{36,37}$. This well explains the coexistence of the PBG and the polarization-dependent attenuation effect. 


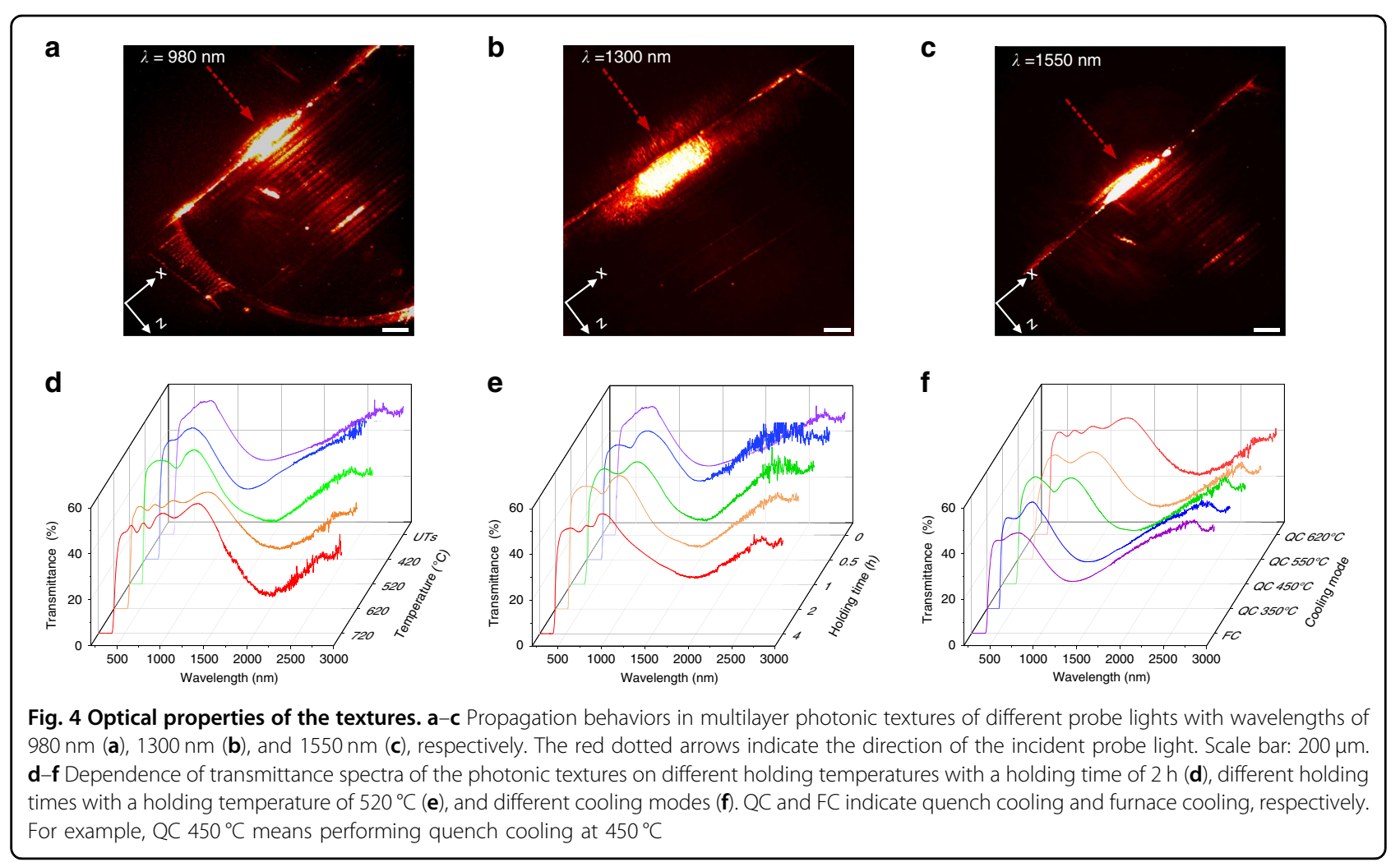

We demonstrated effective tailoring of the optical properties of the SOPTL-fabricated photonic textures. As an example, introducing a reversible secondary-phase transition of the crystal zones in the textures allows for tuning the refractive index contrast between the amorphous phase and the crystal, providing simple and facile access to manipulate optical performances of the photonic textures. Herein, a heat treatment is adopted to induce phase transition along with a quenching process to retain the high-temperature phase. Figure $4 \mathrm{~d}$ indicates that the PBG central wavelength of the texture significantly redshifts from $\sim 1300$ to $\sim 2000 \mathrm{~nm}$ with the temperature increasing from 420 to $720^{\circ} \mathrm{C}$. Notably, no clear difference is found in the transmittance spectra of the initial untreated sample (denoted as UTs) and that treated at $420^{\circ} \mathrm{C}$, indicating that no structural variation occurs in the crystal regions during heat treatment at $420^{\circ} \mathrm{C}$. Figure $4 \mathrm{e}$ demonstrates that an increase in holding time at a fixed temperature of $520^{\circ} \mathrm{C}$ can induce a redshift in the PBG, which is in line with the reported secondary-phasetransition temperature ${ }^{38,39}$. This structural variation is further confirmed by Raman spectroscopy (Supplementary Fig. S6). Similar to the PBG, the polarizationdependent attenuation of the photonic textures is broadly manipulated by the heat treatment process. With an increase in the holding temperature, the wavelength with the maximum attenuation ratio red-shifts from $\sim 1100$ to $\sim 2000 \mathrm{~nm}$ (Supplementary Fig. S7b). Since the secondary-phase transition is reversible ${ }^{38}$, the tuned optical properties of the photonic textures can be easily reset (Fig. 4f). This can be demonstrated by a two-step process that combines furnace cooling and quench cooling, as quench cooling retains the high-temperature phase and furnace cooling gives the room-temperature phase. For example, after $2 \mathrm{~h}$ of heat treatment at $620^{\circ} \mathrm{C}$, the temperature slowly decreases to the set value $\left(T_{\mathrm{f}}\right)$ with furnace cooling, which is followed by quench cooling to room temperature. For the samples with $T_{\mathrm{f}}$ of $550^{\circ} \mathrm{C}$ and $450{ }^{\circ} \mathrm{C}$, blue-shifts of $\sim 200 \mathrm{~nm}$ and $400 \mathrm{~nm}$ to a new waveband in the PBG can be identified, respectively, in comparison with the sample quenched directly from $620^{\circ} \mathrm{C}$. A complete furnace cooling can turn the PBG of a heat-treated sample to the initial state. These results demonstrate that phase-transition engineering serves as a simple and effective method to adjust and reset the optical performances of the photonic textures in a considerable range, making the SOPTL-created textures much more flexible and advantageous over conventional photonic structures.

The processing mechanism of the SOPTL can be further developed to realize more complicated structural tailoring of the photonic textures. For instance, according to the theoretical model of the SOPTL discussed above, the orientation of the periodic arrays depends on the wave vector of the incident light, which has a component in the scanning direction. This indicates that the texture is 

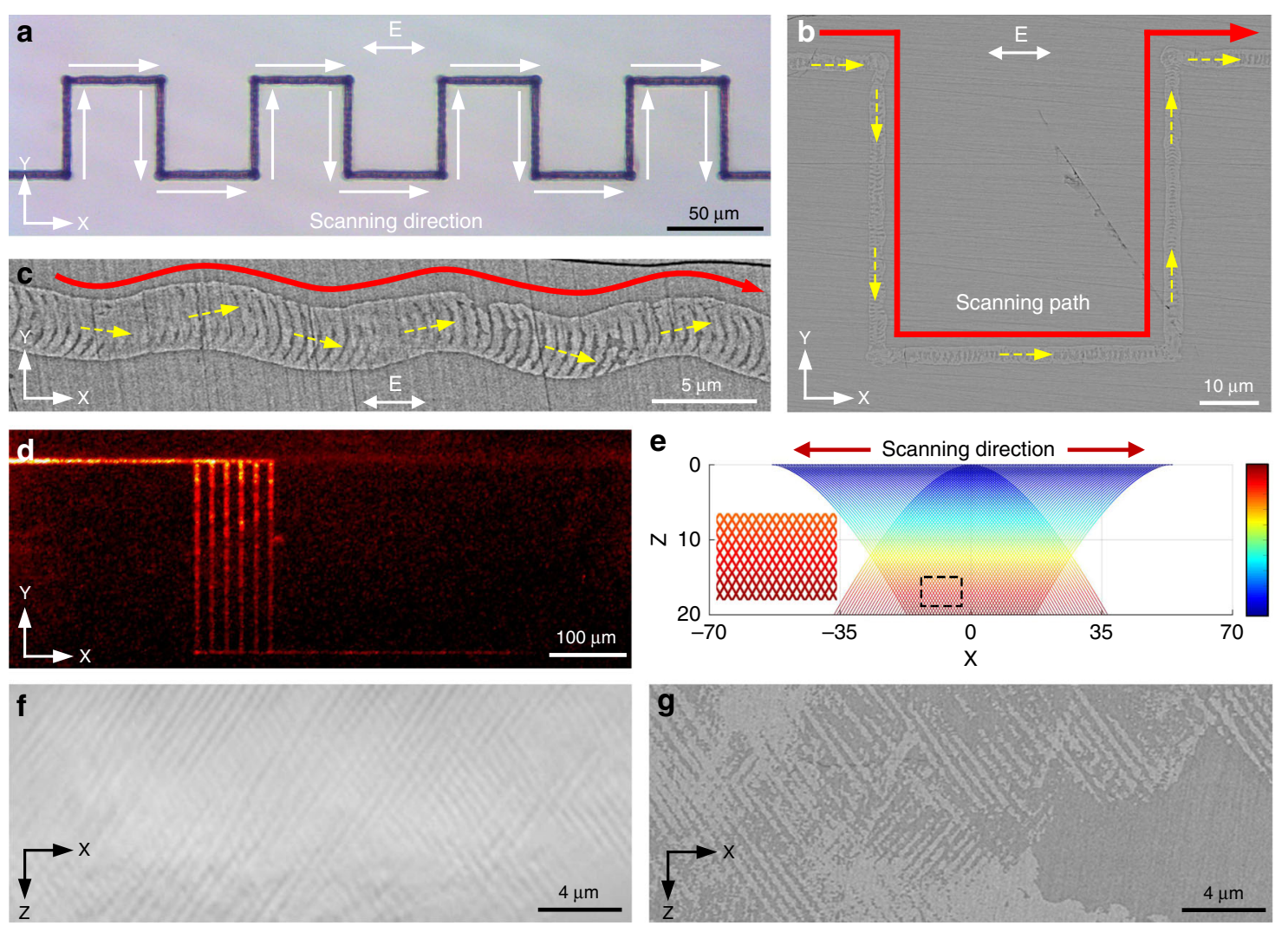

Fig. 5 Demonstration of more complex textures. a Optical image of the line created by the noncontinuous variable-direction writing process. The direction changes $90^{\circ}$ each time. $\mathbf{b}$ Corresponding SEM image of the textures in the scanning path presented in $\mathbf{a}$, and $\mathbf{c}$ shows an SEM image of the textures induced by the continuous variable-direction writing process. The red line indicates the scanning path and the yellow dotted arrow indicates the orientation of the textures. The laser polarization is denoted by E. $\mathbf{d}$ Demonstration of waveguides made of the textures using a 980-nm laser. e Theoretical morphology of the interlaced texture predicted by the SOPTL principle. Inset: the amplified image of the dotted area. The color bar illustrates the radius change of the EPSs. $\mathbf{f}$ Optical microscope image of the interlaced textures created by a dual writing process. $\mathbf{g}$ SEM image of the interlaced photonic textures

scanning-direction-dependent and confers the structure another manipulation degree of freedom. As shown in Fig. 5a, b, in the 90-degree variable-direction writing process, the orientation of the texture only changes with the direction and is independent of the laser polarization (Supplementary Fig. S4b). Further continuous variabledirection writing experiments confirm that such scanning-direction-dependent texture can automatically and continuously adjust its orientation according to the scanning path ( $X Y$ plane, Fig. 5 c), which is highly valuable for constructing spatial photonic crystal elements. For instance, it can be applied to form photonic waveguides to allow the light with wavelengths out of the PBG to travel in the designated paths. Figure $5 \mathrm{~d}$ reveals that the light signal $(980 \mathrm{~nm})$ can survive after passing twelve right angles, indicating excellent light-guiding performance. In addition, the textures with different inclinations can theoretically be superposed together by scanning the ultrafast laser beam back and forth (a dual writing process), resulting in interlaced photonic textures in the glass
(Fig. 5e). This is well demonstrated by experiments. Figure $5 \mathrm{f}$, g demonstrates the formation of a textured nanostructure that consists of two sets of interlaced periodic structures tilted in different directions and this is nearly impossible to achieve by any other approaches. The periodic crystal-glass textures in both $X$ and $Z$ axes can serve as $2 \mathrm{D}$ PCs working in the near-infrared region. In principle, embedded 3D PCs can be further realized by a multilayer SOPTL process.

\section{Discussion}

Controlling the interference-induced periodic crystallization behavior is the intrinsic mechanism of the SOPTL. Therefore, manipulating the laser parameters and the chemical composition of the glass matrix holds the potential to tune the structural and optical properties of the photonic textures. For example, our experiments indicate that the inclination degree of the crystal-glass arrays can be manipulated by adjusting the numerical aperture of the objective (Supplementary Fig. S8a, b). 
Pulse energy also has an effect on the spacing of the ripples. This effect is reflected in the degree of crystallization of the glass matrix. As higher laser fluence will lead to a stronger thermal effect in the interference field, the spacing of the ripples may shrink or even disappear, which is firmly confirmed by our experiments (Supplementary Fig. S8d). Besides, by gradually increasing $\mathrm{Ta}_{2} \mathrm{O}_{5}$ content in the LTN glass, the PBG can be considerably enlarged and becomes much more visible (Supplementary Fig. S7a). Therefore, properly designing laser parameters and glass composition used for the SOPTL offers a direct and efficient way to refine the optical characteristics of the texture.

More importantly, we must emphasize that the proposed physical model can be applied to other glass/crystal materials and similar periodic structures have been successfully created in several commonly used transparent dielectrics, such as fused silica, quartz, and sapphire, which has been confirmed by our experiments (Supplementary Fig. S9). This indicates that the proposed physical model possesses a considerable degree of universality and can serve as a general principle for fabricating allinorganic photonic structures in different transparent media. In addition, the produced textures are well protected by the glass/crystal matrix around, which makes them possess a remarkably long service life and are highly immune to external environmental disturbances such as heat, pressure, moisture, and pollution ${ }^{40,41}$.

In conclusion, the reported 3D interference actively induced by ultrafast laser-matter interaction in the focal area presents a novel optical field modulation and a universal super-resolution tool to produce complicated photonic textures in transparent dielectrics using a single step, breaking through the technical hurdles in achieving single-beam interference lithography. The proposed scattering-interference model provides convincing descriptions to all experimental phenomena and further predicts important properties of the SOPTL process, which allows for the construction of more complicated 3D photonic structures and control over their structural features. The generated photonic textures possess a broad PBG in the near-infrared region, which can be tuned in a wide range by engineering a secondary-phase transition. More importantly, only a few seconds are required to fabricate large-area photonic textures with a nanometerlevel resolution, indicating the promise of the SOPTL in overcoming the bottleneck in the cost and efficiency faced by current nanofabrication techniques. It would be exciting to combine the current laser processing techniques with SOPTL to develop a general technique to achieve in-chip photonic devices at the on-demand position in transparent dielectrics, which will open up brandnew routes for a variety of frontier photonic technologies, including asymmetric gratings, environmental sensors, photonic crystals, dispersion elements, and nonlinear optics.

\section{Materials and methods \\ Materials}

In this study, the textures were created in a multicomponent LTN glass, which is made from a mixture of lanthanum oxide, niobium oxide, and tantalum oxide raw powders at a ratio of $35 \mathrm{La}_{2} \mathrm{O}_{3}-x \mathrm{Ta}_{2} \mathrm{O}_{5}-(65-x) \mathrm{Nb}_{2} \mathrm{O}_{5}$ (where $x=0,15,25$, and $35 \mathrm{~mol} \%$ ). The glass for the SOPTL was prepared by a containerless process where two $\mathrm{CO}_{2}$ lasers were used to melt the mixture of raw materials levitated by the $\mathrm{O}_{2}$ airflow. Before the SOPTL, the blank glass samples were milled into sheets and polished.

\section{Ultrafast laser processing}

In the ultrafast beam-writing process, a mode-locked regeneratively amplified $\mathrm{Yb}: \mathrm{KGW}$-based ultrafast laser system (PHAROS, Light Conversion Ltd.) operating at a wavelength of $1030 \mathrm{~nm}$ with a pulse energy of $1 \mu \mathrm{J}$, a pulse duration of $1 \mathrm{ps}$, and a repetition rate of $150 \mathrm{kHz}$ was employed as the light source. Generally, the laser beam was tightly focused $\sim 60 \mu \mathrm{m}$ below the surface of the sample via a $\times 50$ objective lens $(\mathrm{NA}=0.8)$. In multilayer SOPTL, the processing depth can be up to $\sim 500 \mu \mathrm{m}$. The beam writing was performed by moving the samples at a speed of $2 \mathrm{~mm} \mathrm{~s}^{-1}$, and the highest scanning speed can be up to $6 \mathrm{~mm} \mathrm{~s}^{-1}$. The process window for the SOPTL process is shown in Supplementary Fig. S1b. The movement of the samples in $X Y Z$ axes was realized by a computer-controlled micropositioning platform (Supplementary Fig. S1a).

\section{Testing methodology}

Optical microscopy was performed by a polarizing microscope. The structural properties of the samples were examined by field-emission scanning electron microscopy (FE-SEM), using backscattering mode. For the FE-SEM observation, the textures were polished so as to expose the laser-irradiated region to air and the polished surface was then etched by hydrofluoric acid to improve the contrast of FE-SEM images. Further structural characterization of the created texture was performed by transmission electron microscopy (TEM). The transmittance spectra of samples were measured using a UV-visible NIR spectrophotometer and the secondary-phase transition in the textures was demonstrated by a laser confocal microRaman (LCM-Raman) spectrometer excited by a 532-nm diode-pumped solid-state (DPSS) laser. The heat treatment process was performed in a tube furnace.

Experimental characterization of the light attenuation effect of the textures was performed by illuminating the sample along the $Z$ axis with probe lights that have different polarizations and wavelengths and measuring the 
variation-transmitted light power (Supplementary Fig. S1c). The glass sample was filled with the lines that consist of the textures. The interval of the beam-writing paths is $\sim 15 \mu \mathrm{m}$. The near-infrared light propagation behavior in the textures was imaged using a thermoelectrically cooled InGaAs camera sensitive in the wavelength range $0.9-1.7 \mu \mathrm{m}$.

\section{Acknowledgements}

This work was financially supported by the National Natural Science Foundation of China (Grant Nos. U20A20211, 51902286, 61775192, 61905215, and 51772270), Open funds of the State Key Laboratory of High Field Laser Physics, Shanghai Institute of Optics and Fine Mechanics, Chinese Academy of Sciences, and the Fundamental Research Funds for the Central Universities.

\section{Author details}

${ }^{1}$ State Key Laboratory of Modern Optical Instrumentation, College of Optical Science and Engineering, Zhejiang University, 310027 Hangzhou, China. ${ }^{2}$ School of Materials Science and Engineering, Zhejiang University, 310027 Hangzhou, China. ${ }^{3}$ Centre for Artificial-Intelligence Nanophotonics, School of Optical Science and Engineering, Shanghai University of Science and Technology, 200093 Shanghai, China. ${ }^{4}$ CAS Center for Excellence in Ultraintense Laser Science, Chinese Academy of Sciences, 201800 Shanghai, China

\section{Author contributions}

B.Z., D.T., and J.Q. conceived the idea and initiated the research. J.Q. supervised the project. B.Z. and D.T. designed the experiments. B.Z., D.T., and Z.W. performed the experiments, collected the data, and analyzed the data. B.Z. D.T., Z.W., X.L., B.X., and J.Q. discussed the results. B.Z. and D.T. wrote the paper. M.G., L.T., and J.Q. revised and commented on it.

\section{Conflict of interest}

The authors declare no competing interests.

Supplementary information The online version contains supplementary material available at https://doi.org/10.1038/s41377-021-00534-5.

Received: 9 November 2020 Revised: 27 March 2021 Accepted: 12 April 2021

Published online: 29 April 2021

\section{References}

1. Campbell, M. et al. Fabrication of photonic crystals for the visible spectrum by holographic lithography. Nature 404, 53-56 (2000).

2. Vorobyev, A. Y. \& Guo, C. L. Direct femtosecond laser surface nano/microstructuring and its applications. Laser Photonics Rev. 7, 385-407 (2013).

3. Bonse, J. et al. Laser-induced periodic surface structures-a scientific evergreen. IEEE J. Sel. Top. Quantum Electron. 23, 9000615 (2017).

4. Surdo, S. \& Duocastella, M. Fast acoustic light sculpting for on-demand maskless lithography. Adv. Sci. 6, 1900304 (2019).

5. Zou, T. T. et al. High-speed femtosecond laser plasmonic lithography and reduction of graphene oxide for anisotropic photoresponse. Light.: Sci. Appl. $\mathbf{9}$, 69 (2020).

6. Abid, M. I. et al. Angle-multiplexed optical printing of biomimetic hierarchical 3D textures. Laser Photonics Rev. 11, 1600187 (2017).

7. Ullal, C. K. et al. Photonic crystals through holographic lithography: simple cubic, diamond-like, and gyroid-like structures. Appl. Phys. Lett. 84, 5434-5436 (2004).

8. Maldovan, M. et al. Sub-micrometer scale periodic porous cellular structures: microframes prepared by holographic interference lithography. Adv. Mater. 19, 3809-3813 (2007).

9. Luo, X. G. \& Ishihara, T. Surface plasmon resonant interference nanolithography technique. Appl. Phys. Lett. 84, 4780-4782 (2004).
10. Wang, L. et al. Plasmonic nano-printing: large-area nanoscale energy deposition for efficient surface texturing. Light.: Sci. Appl. 6, e17112 (2017).

11. Öktem, B. et al. Nonlinear laser lithography for indefinitely large-area nanostructuring with femtosecond pulses. Nat. Photonics 7, 897-901 (2013).

12. Rudenko, A., Colombier, J. P. \& Itina, T. E. From random inhomogeneities to periodic nanostructures induced in bulk silica by ultrashort laser. Phys. Rev. B 93, 075427 (2016)

13. Shimotsuma, $Y$. et al. Ultrafast manipulation of self-assembled form birefringence in glass. Adv. Mater. 22, 4039-4043 (2010).

14. Sugioka, K. \& Cheng, Y. Ultrafast lasers-reliable tools for advanced materials processing. Light.: Sci. Appl. 3, e149 (2014).

15. Tan, D. Z. et al. Femtosecond laser induced phenomena in transparent solid materials: fundamentals and applications. Prog. Mater. Sci. 76, 154-228 (2016)

16. Saha, S. K. et al. Scalable submicrometer additive manufacturing. Science $\mathbf{3 6 6}$ 105-109 (2019).

17. Salter, P. S. \& Booth, M. J. Adaptive optics in laser processing. Light.: Sci. Appl. 8, 110 (2019).

18. Joannopoulos, J. D., Villeneuve, P. R. \& Fan, S. H. Photonic crystals: putting a new twist on light. Nature 386, 143-149 (1997).

19. Turner, M. D. et al. Miniature chiral beamsplitter based on gyroid photonic crystals. Nat. Photonics 7, 801-805 (2013).

20. Wu, S. L. et al. Manipulating luminescence of light emitters by photonic crystals. Adv. Mater. 30, 1803362 (2018).

21. Zhang, Q. M. et al. Artificial neural networks enabled by nanophotonics. Light. Sci. Appl. 8, 42 (2019).

22. Krauss, T. F. \& De La Rue, R. M. Photonic crystals in the optical regime-past, present and future. Prog. Quantum Electron. 23, 51-96 (1999).

23. Smith, D. R., Pendry, J. B. \& Wiltshire, M. C. K. Metamaterials and negative refractive index. Science 305, 788-792 (2004).

24. Ródenas, A. et al. Three-dimensional femtosecond laser nanolithography of crystals. Nat. Photonics 13, 105-109 (2018).

25. Noda, S. et al. Full three-dimensional photonic bandgap crystals at nearinfrared wavelengths. Science 289, 604-606 (2000).

26. Arsenault, A. C. et al. From colour fingerprinting to the control of photoluminescence in elastic photonic crystals. Nat. Mater. 5, 179-184 (2006).

27. MacLeod, J. \& Rosei, F. Sustainable sensors from silk. Nat. Mater. 12, 98-100 (2013).

28. Sun, H. B., Matsuo, S. \& Misawa, H. Three-dimensional photonic crystal structures achieved with two-photon-absorption photopolymerization of resin Appl. Phys. Lett. 74, 786-788 (1999).

29. Deubel, M. et al. Direct laser writing of three-dimensional photonic-crystal templates for telecommunications. Nat. Mater. 3, 444-447 (2004).

30. Aoki, K. et al. Microassembly of semiconductor three-dimensional photonic crystals. Nat. Mater. 2, 117-121 (2003).

31. Birowosuto, M. D. et al. Movable high-Q nanoresonators realized by semiconductor nanowires on a Si photonic crystal platform. Nat. Mater. 13, 279-285 (2014)

32. Fleming, J. G. et al. All-metallic three-dimensional photonic crystals with a large infrared bandgap. Nature 417, 52-55 (2002).

33. Hossain, M. M. \& Gu, M. Fabrication methods of $3 D$ periodic metallic nano/ microstructures for photonics applications. Laser Photonics Rev. 8, 233-249 (2014).

34. Kazansky, P. G. et al. Anomalous anisotropic light scattering in Ge-doped silica glass. Phys. Rev. Lett. 82, 2199-2202 (1999).

35. Gattass, R. R. \& Mazur, E. Femtosecond laser micromachining in transparent materials. Nat. Photonics 2, 219-225 (2008).

36. Lalanne, P. \& Lemercier-lalanne, D. On the effective medium theory of subwavelength periodic structures. J. Mod. Opt. 43, 2063-2085 (1996).

37. Zhang, F. et al. Wavelength response and thermal stability of embedded nanograting structure light attenuator fabricated by direct femtosecond laser writing. Appl. Phys. B. 117, 53-58 (2014).

38. Jian, L. \& Wayman, C. M. Monoclinic-to-tetragonal phase transformation in a ceramic rare-earth orthoniobate, $\mathrm{LaNbO}_{4}$. J. Am. Ceram. Soc. 80, 803-806 (1997).

39. Vullum, F. et al. Solid solubility and phase transitions in the system LaNb ${ }_{-x} \mathrm{Ta}_{x} \mathrm{O}_{4}$. J. Solid State Chem. 181, 2580-2585 (2008).

40. Zhang, J. Y. et al. Seemingly unlimited lifetime data storage in nanostructured glass. Phys. Rev. Lett. 112, 033901 (2014)

41. Huang, X. J. et al. Reversible 3D laser printing of perovskite quantum dots inside a transparent medium. Nat. Photonics 14, 82-88 (2020). 